\title{
Functional analysis of evolutionary human methylated regions in schizophrenia patients
}

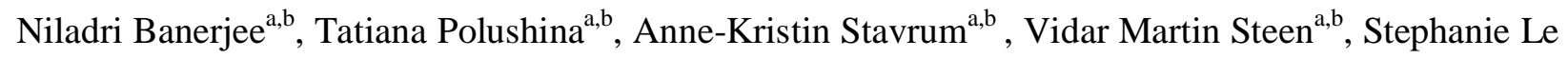
Hellard $^{\mathrm{a}, \mathrm{b}}$

a. NORMENT, Department of Clinical Science, University of Bergen, Bergen, Norway

b. Dr. Einar Martens Research Group for Biological Psychiatry, Department of Medical Genetics, Haukeland University Hospital, Bergen, Norway

Correspondence to: Prof. Stéphanie Le Hellard, Department of Clinical Medicine, Laboratory Building, Haukeland University Hospital, N-5021 Bergen, Norway.

Telephone: +47-559753 37

Fax: +47-559754 79

Email: stephanie.hellard@uib.no

\section{Authors' email addresses}

Niladri Banerjee

Tatiana Polushina

Anne-Kristin Stavrum

Vidar M. Steen

Stéphanie Le Hellard niladri.banerjee@uib.no

tatiana.polushina@uib.no

anne-kristin.stavrum@uib.no

vidar.martin.steen@helse-bergen.no

stephanie.hellard@uib.no 


\begin{abstract}
Background: Recent studies have implicated variations in DNA methylation in the aetiology of schizophrenia. Genome-wide scans in both brain and blood report differential methylated regions (DMRs) and positions (DMPs) between patients with schizophrenia and healthy controls. Previously, we reported that DMRs where human specific methylation (hDMR) has occurred over evolutionary time are enriched for schizophrenia-associated markers (SCZ_hDMR). However, it is unknown whether these human specific DMRs show variable methylation in patients with schizophrenia.

Methods: Using publicly available data, we investigate if human specific DMRs that harbour genetic variants associated with schizophrenia are differentially methylated between cases and controls.

Results: We find statistically significant $(p<1 \mathrm{e}-4)$ methylation difference in schizophrenia associated human specific DMRs (SCZ hDMR) between brain samples of cases and controls. However, we fail to find evidence of similar differences in methylation in blood samples.

Conclusion: Regions that are evolutionarily important for human species and that are associated with schizophrenia, also show difference in methylation variation in the brain in patients with schizophrenia.
\end{abstract}




\section{Introduction}

Schizophrenia is a devastating psychiatric illness that has a global lifetime prevalence of $1 \%$ [1] and affects patients in the prime of their youth. It has no cure and treatment relies on antipsychotic drugs that can cause several side effects such as weight gain [2], obesity [3] and metabolic syndrome [4]. These side effects are believed to induce higher mortality amongst patients with schizophrenia with average life expectancy reducing by nearly 20 years $[5,6]$.

Much research has been undertaken in the last decade to unravel the genetics of the disease in the hope of finding better treatment options for the patients. Research has revealed the disease to have complex aetiology with the latest genome-wide association study (GWAS) reporting over a hundred loci genome-wide [7]. While these results have brought more understanding about the regions involved in the disease, much work remains to elucidate the biological mechanisms. A majority of the genomewide hits are in the non-coding regions [7] and efforts are on to investigate the role of epigenetic factors in these regions [8]. For instance, differences in DNA methylation pattern amongst patients with schizophrenia, compared to healthy controls at both candidate gene loci [9-12] and at the global genome-wide level [13-17] have been reported.

DNA methylation has also been investigated in studies of epigenetic inheritance [18-20] and paleoepigenetics [21-23]. For example, Gokhman and colleagues [23] looked at methylation differences between humans and our extinct hominid cousins- Neanderthals and Denisovans. Although most of the methylated regions were similar between the three hominids, 2000 regions were differentially methylated amongst the three hominid species. Building up on their study, we found that the human specific differentially methylated regions (hDMRs) show an enrichment of genetic variants associated 
with schizophrenia (compared to the rest of the genome [24]). While regions differentially methylated between human and great apes [25] do not show such an enrichment.

Other genetic studies have also suggested that genomic markers of recent evolution are enriched for schizophrenia associated single nucleotide polymorphisms (SNPs ) [28-30]. Thus together with our results this has narrowed the evolutionary hypothesis of the schizophrenia proposed by TJ Crow and others $[26,27]$ to a possible effect of recent evolution in the maintenance of schizophrenia in humans.

We have now investigated whether these human specific DMRs which are in regions of genetic association with for schizophrenia could also show differences in methylation between patients with schizophrenia and controls. 


\section{Materials \& Methods}

\section{Evolutionary Differentially Methylated Region (DMR) data}

Gokhman et al. [23] published a list of DMRs they term as human-specific differentially methylated regions (hDMRs). They identified these DMRs by comparing human methylome against Neanderthal and Denisovan methylomes. They represent human lineage specific methylation markers since the divergence of the common ancestor of Neanderthals and Denisovans.

\section{Evolutionary DMR selection for analysis}

We have previously reported the enrichment of association of the hDMRs for schizophrenia [24]. Among the 891 hDMRs, we focus on DMRs containing SNPs significantly associated with schizophrenia. We first determine an experiment-wide significance threshold to select the SNPs and DMRs. About 27,000 SNPs were located in or in linkage-disequilibrium (LD) with h_DMRs [24]. We followed the procedure of Moskvina et al. [31] and estimated 2048 independent SNPs that with a Bonferroni correction at $\alpha=0.05$ gives an experiment-wide threshold of 2.46x10e-5. Nine DMRs were found to harbour SNPs that passed this threshold and were carried forward in the analysis DMR10, DMR127, DMR203, DMR204, DMR236, DMR237, DMR291, DMR526, DMR527 (Additional File 1, 2) To account for the sparse and irregular coverage of array probes in these DMRs, we included the $\sim 3 \mathrm{kbp}$ flanking regions of the 9 DMRs, which gave a final list of 65 probes that covered a maximum these 9 DMRs (Additional File 1).

\section{Brain and Blood Methylation Data}

Data for methylation variation in brain were obtained from publicly available datasets at GEO accession numbers : GSE61107 [14], GSE6143 [16], GSE61380 [16], GSE74193 [17] and for blood at 
GSE80417 [15]. Normalized beta matrix was used for all datasets except for GSE61107 where raw IDAT files were available.

\section{Data Processing}

All the publicly available methylation data analysed in this study were obtained from the Illumina HumanMethylation450 BeadChip platform. IDAT files were available for the study by Wockner and colleagues [14]. We removed probes that failed at a detection $p$-value of 0.05 in $50 \%$ of the samples. Subsequently, functional normalization [32] was performed using the minfi [33] package in R [34]. During pre-processing we found that 4 DMRs had only a single overlapping probe. (Additional File 1). These probes are also in the list from Zhou et al. [35] where they recommend removing the probes as they contain SNPs that could interfere with probe performance (Additional File 1). We chose not to remove these probes, as doing so would eliminate the only methylation signal within the DMRs. Details of the other DMRs containing probes suggested for masking are available in additional file 1. Similarly, we did not need to remove probes on the sex chromosomes since none of the evolutionary DMRs selected were on these chromosomes.

The study by Pidsley et al. [16] comprised of two different brain datasets obtained from the Douglas Bell Canada Brain Bank (DBCBB) and London Neurodegenerative Diseases Brain Bank (LNDBB) . These were analysed separately similar to a comparative analyses performed by Wockner and colleagues [13].

The study by Jaffe et al. [17] initially comprised of more than 650 samples in total. After removing duplicate and pre-natal data, we focused on a final set of 191 schizophrenia patient samples with 231 controls with a similar age distribution (Supplementary Table T1).

Normalized data from Pidsley et al., 2014 [16] and Hannon et al., 2016 [15] were used directly. 


\section{Visualization of DMRs}

DMRs were visualized using the Gviz package [36] in R [34]. Genomic coordinates for SNPs and RefSeq genes in hg19 were obtained from the UCSC Table Browser [37]. CpG island information was obtained using the Annotation Hub package [38]. Probe annotation for the Illumina HumanMethylation450 BeadChip was obtained from study by Zhou et al. [35].

\section{Significance testing of DMPs and DMRs}

We implemented pipeline using limma [39,40] package in $\mathrm{R}$ [34] with empirical Bayes [41] to determine the differentially methylated positions (DMPs) that showed statistically significant differences in methylation between patients with schizophrenia and controls. Details of the linear models implemented for each of the datasets may be found in supplementary file.

To determine whether the specific evolutionary DMRs selected showed statistically significant differences in methylation at the region level, we implemented comb-p [42], which combines adjacent $p$-values and performs false discovery rate adjustment. This makes it ideal for analysing data from DNA methylation arrays where probes are irregularly spaced. Furthermore, comb-p allows one to test custom defined regions for statistical significance. In our case, we implemented comb-p for the individual DMRs. We report here the $p$-values from one-step Sidak multiple testing correction performed using the region_p programme.

\section{Epigenetic Annotation}

The 18-state chromatin annotation was obtained from the Roadmap Epigenome project [43] for sample E073 that contains a mixture of dorso-lateral prefrontal cortex tissues from two people aged 75 and 81 . Additionally, chromatin state annotation was obtained from peripheral blood mononuclear cells from 
sample E062. The BED file containing the genome coordinates of the 18 state model was intersected with the coordinates of the 9 DMRs in R [34] using the Genomic Ranges package [44].

\section{Results}

Evolutionary enriched DMRs show methylation variation in patients with schizophrenia

We utilised the brain methylation datasets from studies conducted by Wockner et al.. [14] $(\mathrm{N}=48,24$ cases with schizophrenia, 24 controls), Pidsley et al.. (LNDBB: $\mathrm{N}=46,22$ cases with schizophrenia and 24 controls; DBCBB: $\mathrm{N}=33,18$ cases with schizophrenia and 15 controls) [16] and Jaffe et al. ( $\mathrm{N}$ $=322,191$ cases with schizophrenia and 231 controls) [17]. All three studies utilized the prefrontal cortex for methylation analyses. We find evidence of variable methylation in the SCZ_hDMRs in all three datasets.

In the largest brain methylation dataset from Jaffe et al. [17], we find methylation differences between cases and controls for DMR203 $(p=7.29 \mathrm{e}-17)$ (Figure 1), DMR291 $(p=1.756 \mathrm{e}-4)$ and DMR526 $(p=$ 1.019e-4) (Table 1). This dataset might be more reliable due to all the factors that could be included in the regression model which is not the case for other datasets (Supplementary Methods ). DMR203 also shows different level of methylation between cases and controls in the DBCBB dataset from Pidsley et al. (Supplementary Figure S1, Table 1). DMR127 shows differences in methylation in the brain datasets from Wockner (Figure 2) and Pidsley (Supplementary Figure S2, S3), but the direction of effect is opposite in the two samples. Furthermore, although DMR127 replicates in both the LNDBB and DBCBB datasets from Pidsley, it survives multiple testing correction only in the LNDBB dataset (Table 1) 
We performed the same analyses in blood samples obtained from Hannon et al. [15] $(\mathrm{N}=675,353$ cases with schizophrenia and 322 controls). We found no evidence of different methylation in any of the SCZ_hDMRs in blood samples (Supplementary Figures S4, S5, Supplementary Table T2). However, our analysis was limited due to lack of information on all the covariates from the Hannon study [15].

\section{Evolutionary enriched DMRs occur in diverse genome annotations}

We annotated the evolutionary enriched DMRs using data from the Roadmap Epigenomics Consortium [43]. We utilised the 18-state chromatin model from sample E073 [17] that comprises of data from a mixture of the dorsolateral prefrontal cortex from two persons aged 75 and 81 years old. The annotation suggests DMRI27 to be present in an active enhancer that overlaps LINC0606; DMR203 overlaps an active transcription start site for ZSCAN12P1 (Supplementary Table T3). Additionally, we find that DMR291 overlaps weak enhancers in FGFRI (Supplementary Table T3). In addition, using Hi-C data from the hippocampus [45] in the 3D Genome Browser [46] we observed that DMR526 is potentially located in a regulatory region for the gene MARK3 (Supplementary Figure S6) . We also annotate the DMRs using the chromatin state data in blood from E062. We find that the DMRs that are significantly differentially methylated in the brain datasets occur in heterochromatin regions in blood (Supplementary Table T3). 


\section{Discussion \& Conclusion}

We report here the investigation of DNA methylation differences between cases with schizophrenia and controls in regions that show differential methylation between modern humans and Neanderthal/Denisovan and which are associated with schizophrenia. We observe the difference in methylation for several of these regions in brain samples but not in blood samples.

DMR203 shows statistically significant methylation difference in patients with schizophrenia compared to controls that survives multiple testing correction using the Sidak procedure in comb-p. We find evidence in the largest set of brain samples from Jaffe et al. [17] ( $p<1 \mathrm{e}-16$, Sidak correction) and the DBCBB samples ( $p<0.05$, Sidak correction) from Pidsley et al. [16]. However, the direction of effect is not consistent. This could be due to lack of power in the two samples (type I error in both), however it could also be due to different cell composition between the two studies. We observe hypomethylation in samples from Jaffe et al. [17] (Figure 1) and hyper-methylation in the DBCBB samples [16] (Supplementary Figure S1). The DMR203 is within the extended MHC region that is known to harbour the genetic variants with the strongest association with schizophrenia. This DMR overlaps with the pseudo gene ZSCAN12P1. The gene has also been implicated in schizophrenia and autism spectrum disorder in a recent meta-analysis [47].

DMR127 shows statistically significant differences in methylation in both the Wockner dataset [14] and the LNDBB dataset from Pidsley et al.[16], but the direction of effect is opposite between the two studies. We observe hypo-methylation in the schizophrenia samples from Wockner [14] (Figure 2) while the LNDBB samples from Pidsley et al.[16] show hyper-methylation in the patient samples (Supplementary Figure S2). This could again be due to lack of power in the two samples or as reported previously, because of different cell composition between the two studies [13]. Interestingly, DMR127 also shows significantly differentially methylated probes (DMPs) in the DBCBB dataset 
from Pidsley et al. (Supplementary Figure S3), but the region does not survive corrections for multiple testing multiple testing in comb-p (Table 1). The region of DMR127 covers the long intergenic non-protein coding RNA 606, LINC0606. Since there is different methylation in patients with schizophrenia in this region, it is possible that the regulation of this gene is important in the aetiology of schizophrenia. The gene is actively expressed in the brain and occurs upstream of SLC6A11 and downstream of ATP2B2, both of which are also highly expressed in the brain and have been implicated in various neurological disorders such as epilepsy [48], autism [49] and schizophrenia [50]. However, DMR127 does not replicate in the largest brain methylation dataset from Jaffe et al. [17]. It is possible that due to increased sample size and power in the dataset from Jaffe et al. [17], the hypo- and hyper-methylation observed in the smaller datasets of Wockner et al. [14] and the LNDBB dataset [16] get averaged out. There may also be specific sub-populations of patients with schizophrenia who show either hypo or hyper-methylation in this region. The other possibility is the lack of identical cell types analysed across all the brain datasets. This is a challenge for the field of psychiatric genetics that the brain is a heterogeneous tissue filled with varying levels of neurons, glial cells and astrocytes. This makes comparative epigenetics studies difficult to execute and implement.

Finally we find some methylation differences in three additional DMRs $-D M R 237(p<1 \mathrm{e}-2$, Sidak correction ), DMR291 ( $p<1 \mathrm{e}-3$, Sidak correction) and DMR526 ( $p<1 \mathrm{e}-3$, Sidak correction $)$ in the dataset from Jaffe et al. [17]. DMR237 occurs in the intronic region of MAD1L1, but the significance appears to be driven by a single DMP, instead of a broad change across the DMR (data available on request). DMR291 overlaps the gene FGFRI while DMR526 is in a non-coding region of the genome. FGFR1 has been previously implicated in schizophrenia [51,52]. DMR526 does not overlap with any genes but may be involved in long-range regulation of MARK3 determined through Hi-C genome-wide contact matrix (Supplementary Figure S6), 
An important caveat of the present analysis is that many of the DMRs have low probe coverage (Additional File 1). For instance, DMR526 which shows some difference in methylation in the dataset from Jaffe et al. is represented by only one probe. Several other DMRs had limited probe coverage which is a major limitation of our analyses. In the normalized datasets from Pidsley et al. [16], the only probe in DMR526 was missing. This also implies a subsequent limitation of the statistical test in comb$p$. For multiple testing correction using the Sidak procedure, the algorithm relies on probe coverage in the DMRs. Thus, filtering out probes may have an impact on the final $p$-values reported by Sidak correction.

When looking at the blood samples [15] from patients with schizophrenia, we fail to find evidence of any methylation variation in these evolutionary DMRs. This is unlikely due to lack of power as the sample size from Hannon et al. [15] is comparable to that of Jaffe et al. [17]. The lack of replication in blood might instead suggest that the disease is more specific to the brain and thus we may not expect to observe difference in blood, at least in the particular SCZ_hDMRs analysed in the present study. This may be supported by the fact that the 18-state chromatin annotation from REMC for peripheral blood mononuclear cells shows the most significant SCZ_hDMRs to be in quiescent and repressed chromatin regions (Supplementary Table T3). This is supported by data from GTEx [53] that shows higher expression of the genes in the specific SCZ_hDMRs in brain over blood (Supplementary Figure S4, S5). Furthermore, we cannot rule out that there may be other h_DMRs that do not contain significant SCZ SNPs, but may show disrupted methylation variation in blood. Additionally we cannot rule out that the methylation variation observed in brain samples is due to anti-psychotic medications [54]. An additional point to consider is that the variation we observe in the evolutionary DMRs in brain may in fact be driven by methylation quantitative trait loci (mQTL). Such mQTLs may act in cis upto $1 \mathrm{Mb}$ from the $\mathrm{CpG}$ site [55]. Previous research into foetal brain mQTLs has 
demonstrated a significant enrichment with schizophrenia associated regions [56]. Future research should look into the influence of genotypes affecting methylation variation in evolutionary DMRs.

In conclusion, we observe that regions of the human genome whose methylation is specific to human evolution and are enriched for schizophrenia associated markers show disrupted methylation in brain samples of patients with schizophrenia. This disrupted methylation is not observed in blood samples. Our results suggest dysregulation of methylation in novel regions of the genome in patients with schizophrenia in brain tissue. Future research should be carried out with higher probe density in the evolutionary DMR regions or use whole genome methylation sequencing technologies.

\section{References}

[1] Ayuso-Mateos JL. Global Burden of schizophrenia in the year 2000-Version 1 Estimates. World Health Organization 2002.

[2] Rummel-Kluge C, Komossa K, Schwarz S, Hunger H, Schmid F, Lobos CA, et al. Head-to-head comparisons of metabolic side effects of second generation antipsychotics in the treatment of schizophrenia: a systematic review and meta-analysis. Schizophrenia Research 2010;123:225-33.

[3] Baptista T, Kin N, Beaulieu S, De Baptista E. Obesity and related metabolic abnormalities during antipsychotic drug administration: mechanisms, management and research perspectives. Pharmacopsychiatry 2002;35:205-19.

[4] McEvoy JP, Meyer JM, Goff DC, Nasrallah HA, Davis SM, Sullivan L, et al. Prevalence of the metabolic syndrome in patients with schizophrenia: baseline results from the Clinical Antipsychotic Trials of Intervention Effectiveness (CATIE) schizophrenia trial and comparison with national estimates from NHANES III. Schizophrenia Research 2005;80:19-32.

[5] Hjorthøj C, Stürup AE, McGrath JJ, Nordentoft M. Years of potential life lost and life expectancy in schizophrenia: a systematic review and meta-analysis. The Lancet Psychiatry 2017;4:295-301.

[6] Laursen TM. Life expectancy among persons with schizophrenia or bipolar affective disorder. Schizophr Res 2011;131:101-4.

[7] Ripke S, Neale BM, Corvin A, Walters JT, Farh K-H, Holmans PA, et al. Biological insights from 108 schizophrenia-associated genetic loci. Nature 2014;511:421.

[8] O'Dushlaine C, Rossin L, Lee PH, Duncan L, Parikshak NN, Newhouse S, et al. Psychiatric genome-wide association study analyses implicate neuronal, immune and histone pathways. Nature Neuroscience 2015;18:199. 
[9] Fikri RMN, Norlelawati AT, El-Huda ARN, Hanisah MN, Kartini A, Norsidah K, et al. Reelin (RELN) DNA methylation in the peripheral blood of schizophrenia. Journal of Psychiatric Research 2017;88:28-37.

[10] Mitchelmore C, Gede L. Brain derived neurotrophic factor: epigenetic regulation in psychiatric disorders. Brain Research 2014;1586:162-72.

[11] Ikegame T, Bundo M, Sunaga F, Asai T, Nishimura F, Yoshikawa A, et al. DNA methylation analysis of BDNF gene promoters in peripheral blood cells of schizophrenia patients. Neurosci Res 2013;77:208-14.

[12] Abdolmaleky HM, Nohesara S, Ghadirivasfi M, Lambert AW, Ahmadkhaniha H, Ozturk S, et al. DNA hypermethylation of serotonin transporter gene promoter in drug naïve patients with schizophrenia. Schizophr Res 2014;152:373-80.

[13] Wockner L, Morris C, Noble E, Lawford B, Whitehall V, Young R, et al. Brain-specific epigenetic markers of schizophrenia. Translational Psychiatry 2015;5:e680.

[14] Wockner L, Noble E, Lawford B, Young RM, Morris C, Whitehall V, et al. Genome-wide DNA methylation analysis of human brain tissue from schizophrenia patients. Translational Psychiatry 2014;4:e339.

[15] Hannon E, Dempster E, Viana J, Burrage J, Smith AR, Macdonald R, et al. An integrated genetic-epigenetic analysis of schizophrenia: evidence for co-localization of genetic associations and differential DNA methylation. Genome Biology 2016;17:176.

[16] Pidsley R, Viana J, Hannon E, Spiers H, Troakes C, Al-Saraj S, et al. Methylomic profiling of human brain tissue supports a neurodevelopmental origin for schizophrenia. Genome Biology 2014; 15:483.

[17] Jaffe AE, Gao Y, Deep-Soboslay A, Tao R, Hyde TM, Weinberger DR, et al. Mapping DNA methylation across development, genotype and schizophrenia in the human frontal cortex. Nature Neuroscience 2016;19:40.

[18] Klengel T, Dias BG, Ressler KJ. Models of intergenerational and transgenerational transmission of risk for psychopathology in mice. Neuropsychopharmacology 2016;41:219.

[19] Dias BG, Ressler KJ. Parental olfactory experience influences behavior and neural structure in subsequent generations. Nature Neuroscience 2014;17:89-96.

[20] Klosin A, Casas E, Hidalgo-Carcedo C, Vavouri T, Lehner B. Transgenerational transmission of environmental information in C. elegans. Science 2017;356:320-3.

[21] Gokhman D, Meshorer E, Carmel L. Epigenetics: it's getting old. Past meets future in paleoepigenetics. Trends in Ecology \& Evolution 2016;31:290-300.

[22] Gokhman D, Malul A, Carmel L. Inferring Past Environments from Ancient Epigenomes. Molecular Biology and Evolution 2017;34:2429-38. 
[23] Gokhman D, Lavi E, Prüfer K, Fraga MF, Riancho JA, Kelso J, et al. Reconstructing the DNA methylation maps of the Neandertal and the Denisovan. Science 2014;344:523-7.

[24] Banerjee N, Polushina T, Bettella F, Giddaluru S, Steen VM, Andreassen OA, et al. Recently evolved human-specific methylated regions are enriched in schizophrenia signals. BMC Evolutionary Biology 2018;18.

[25] Banerjee N, Polushina T, Bettella F, Steen VM, Andreassen OA, Le Hellard S. Analysis of differentially methylated regions in primates and nonprimates provides support for the evolutionary hypothesis of schizophrenia. bioRxiv 2018:322693.

[26] Crow TJ. The $\square$ big bang $\square$ theory of the origin of psychosis and the faculty of language. Schizophrenia Research 2008;102:31-52.

[27] Crow TJ. Is schizophrenia the price that Homo sapiens pays for language? Schizophr Res 1997;28:127-41.

[28] Srinivasan S, Bettella F, Hassani S, Wang Y, Witoelar A, Schork AJ, et al. Probing the association between early evolutionary markers and schizophrenia. PloS One 2017;12:e0169227.

[29] Xu K, Schadt EE, Pollard KS, Roussos P, Dudley JT. Genomic and network patterns of schizophrenia genetic variation in human evolutionary accelerated regions. Mol Biol Evol 2015;32:1148-60.

[30] Srinivasan S, Bettella F, Mattingsdal M, Wang Y, Witoelar A, Schork AJ, et al. Genetic markers of human evolution are enriched in schizophrenia. Biological Psychiatry 2015.

[31] Moskvina V, Schmidt KM. On multiple-testing correction in genome-wide association studies. Genetic Epidemiology 2008;32:567-73.

[32] Fortin J-P, Labbe A, Lemire M, Zanke BW, Hudson TJ, Fertig EJ, et al. Functional normalization of $450 \mathrm{k}$ methylation array data improves replication in large cancer studies. Genome Biology 2014; 15:503.

[33] Aryee MJ, Jaffe AE, Corrada-Bravo H, Ladd-Acosta C, Feinberg AP, Hansen KD, et al. Minfi: a flexible and comprehensive Bioconductor package for the analysis of Infinium DNA methylation microarrays. Bioinformatics 2014;30:1363-9.

[34] Team RC. R: A Language and Environment for Statistical Computing. Vienna, Austria: 2017.

[35] Zhou W, Laird PW, Shen H. Comprehensive characterization, annotation and innovative use of Infinium DNA methylation BeadChip probes. Nucleic Acids Research 2017;45:e22-e22.

[36] Hahne F, Ivanek R. Visualizing genomic data using Gviz and bioconductor. Statistical Genomics: Methods and Protocols 2016:335-51.

[37] Kent WJ, Sugnet CW, Furey TS, Roskin KM, Pringle TH, Zahler AM, et al. The human genome browser at UCSC. Genome Research 2002;12:996-1006. 
[38] Morgan M. AnnotationHub: Client to access AnnotationHub resources. R package version 2.14.2. 2018 .

[39] Smyth G. limma: Linear Models for Microarray Data. In: Gentleman, Robert and Carey, Vincent J. and Huber, Wolfgang and Irizarry, Rafael A. and Dudoit, Sandrine, editor. Bioinformatics and Computational Biology Solutions Using R and Bioconductor, Springer New York; 2005, p. 397420 .

[40] Ritchie ME, Phipson B, Wu D, Hu Y, Law CW, Shi W, et al. limma powers differential expression analyses for RNA-sequencing and microarray studies. Nucleic Acids Research 2015;43:e47-e47.

[41] Smyth GK. Linear models and empirical bayes methods for assessing differential expression in microarray experiments. Stat Appl Genet Mol Biol 2004;3:Article3.

[42] Pedersen BS, Schwartz DA, Yang IV, Kechris KJ. Comb-p: software for combining, analyzing, grouping and correcting spatially correlated P-values. Bioinformatics 2012;28:2986-8.

[43] Kundaje A, Meuleman W, Ernst J, Bilenky M, Yen A, Heravi-Moussavi A, et al. Integrative analysis of 111 reference human epigenomes. Nature 2015;518:317-30.

[44] Lawrence M, Huber W, Pagès H, Aboyoun P, Carlson M, Gentleman R, et al. Software for computing and annotating genomic ranges. PLoS Comput Biol 2013;9:e1003118.

[45] Schmitt AD, Hu M, Jung I, Xu Z, Qiu Y, Tan CL, et al. A compendium of chromatin contact maps reveals spatially active regions in the human genome. Cell Reports 2016;17:2042-59.

[46] Wang Y, Zhang B, Zhang L, An L, Xu J, Li D, et al. The 3D Genome Browser: a web-based browser for visualizing $3 \mathrm{D}$ genome organization and long-range chromatin interactions. BioRxiv 2017:112268.

[47] Consortium ASDWG of TPG, Anney RJ, Ripke S, Anttila V, Grove J, Holmans P, et al. Metaanalysis of GWAS of over 16,000 individuals with autism spectrum disorder highlights a novel locus at 10q24. 32 and a significant overlap with schizophrenia. Molecular Autism 2017;8:1-17.

[48] Dikow N, Maas B, Karch S, Granzow M, Janssen JW, Jauch A, et al. 3p25. 3 microdeletion of GABA transporters SLC6A1 and SLC6A11 results in intellectual disability, epilepsy and stereotypic behavior. American Journal of Medical Genetics Part A 2014;164:3061-8.

[49] Carayol J, Sacco R, Tores F, Rousseau F, Lewin P, Hager J, et al. Converging evidence for an association of ATP2B2 allelic variants with autism in male subjects. Biological Psychiatry 2011;70:880-7.

[50] Ikeda M, Tomita Y, Mouri A, Koga M, Okochi T, Yoshimura R, et al. Identification of novel candidate genes for treatment response to risperidone and susceptibility for schizophrenia: integrated analysis among pharmacogenomics, mouse expression, and genetic case-control association approaches. Biological Psychiatry 2010;67:263-9.

[51] Stachowiak MK, Birkaya B, Aletta J, Narla S, Benson C, Decker B, et al. Nuclear FGF receptor1 and CREB binding protein: An integrative signaling module. Journal of Cellular Physiology 
2015;230:989-1002.

[52] Narla S, Lee Y, Benson C, Sarder P, Brennand K, Stachowiak E, et al. Common developmental genome deprogramming in schizophrenia-Role of Integrative Nuclear FGFR1 Signaling (INFS). Schizophrenia Research 2017;185:17-32.

[53] Lonsdale J, Thomas J, Salvatore M, Phillips R, Lo E, Shad S, et al. The genotype-tissue expression (GTEx) project. Nature Genetics 2013;45:580.

[54] Connor CM, Akbarian S. DNA methylation changes in schizophrenia and bipolar disorder. Epigenetics 2008;3:55-8.

[55] Zhang D, Cheng L, Badner JA, Chen C, Chen Q, Luo W, et al. Genetic control of individual differences in gene-specific methylation in human brain. The American Journal of Human Genetics 2010;86:411-9.

[56] Hannon E, Spiers H, Viana J, Pidsley R, Burrage J, Murphy TM, et al. Methylation QTLs in the developing brain and their enrichment in schizophrenia risk loci. Nature Neuroscience 2016;19:48. 


\section{Figure and table legends}

Figure 1- Methylation variation in DMR203, in samples from Jaffe et al.

Figure depicts methylation variation in patients with schizophrenia in the evolutionary DMR203 (maroon, $p=$ ) in the prefrontal cortex. Schizophrenia methylation (pink) compared with controls (blue) from Jaffe et al. [17]. Significant differentially methylated positions (DMPs) depicted $* *(p<0.005), * * *(p<0.0005)$, and $* * * *(p<0.00001)$

\section{Figure 2- Methylation variation in DMR127, in samples from Wockner et al.}

Figure depicts methylation variation in patients with schizophrenia in the evolutionary DMR (maroon) in the prefrontal cortex. Schizophrenia methylation (pink) compared with controls (blue) from Wockner et al. [14]. Significant differentially methylated positions (DMPs) depicted as * $(p<0.05), * *(p<0.005)$ and $* * *(p$ $<0.0005)$

\section{Table 1- Comb-p results across all evolutionary DMRs and datasets}

Table depicts the $p$-values from Sidak multiple correction for all the DMRs across all the brain methylation datasets obtained from comb- $p$. Statistically significant DMRs are denoted with $*(p<5 \mathrm{e}-$ 2), $* *(p<1 \mathrm{e}-3)$ and $* * *(p<1 \mathrm{e}-4) . D M R 526$ and DMR527 lacked probes, possibly removed during pre-processing by Pidsley et al. [16] and as such could not be tested. 
bioRxiv preprint doi: https://doi.org/10.1101/540294; this version posted February 5, 2019. The copyright holder for this preprint (which was not certified by peer review) is the author/funder, who has granted bioRxiv a license to display the preprint in perpetuity. It is made available under aCC-BY-NC-ND 4.0 International license.



Figure 1

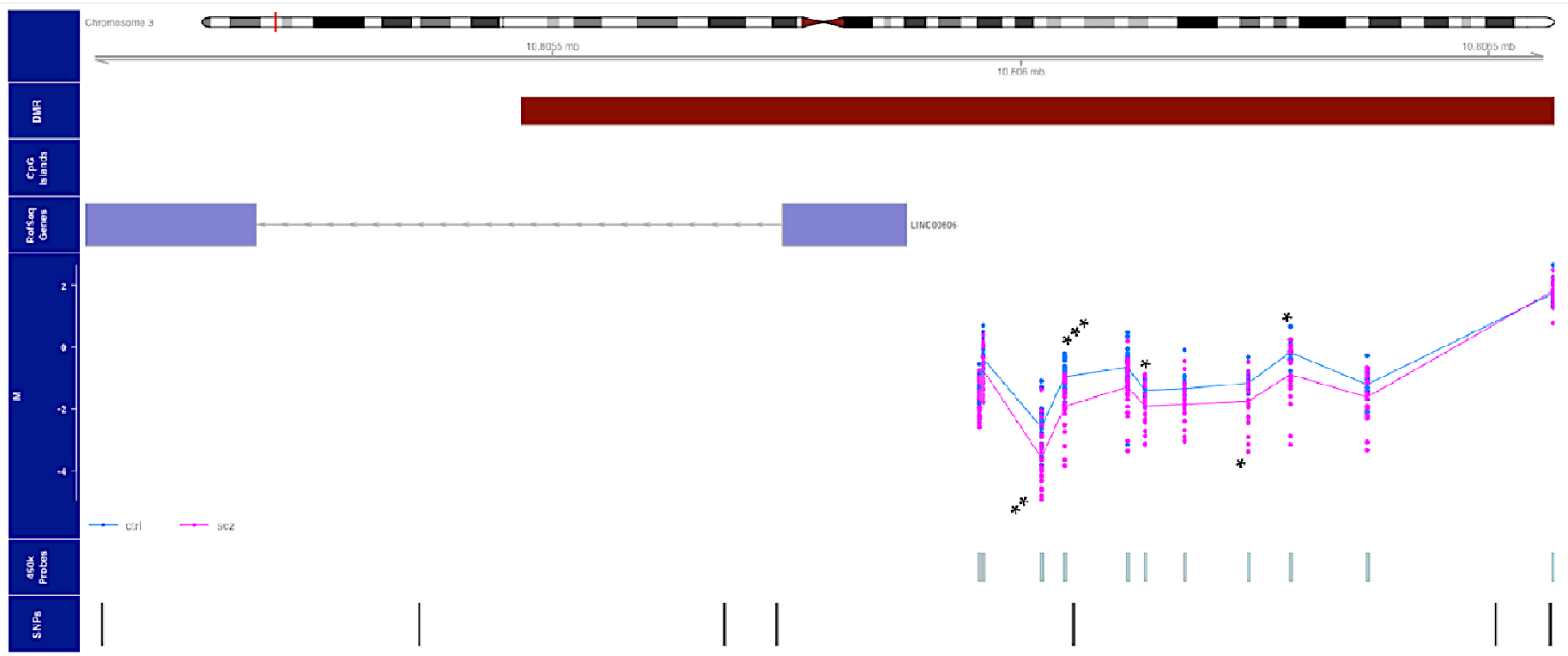

Figure 2 
bioRxiv preprint doi: https://doi.org/10.1101/540294; this version posted February 5, 2019. The copyright holder for this preprint (which was not certified by peer review) is the author/funder, who has granted bioRxiv a license to display the preprint in perpetuity. It is made available under aCC-BY-NC-ND 4.0 International license.

\section{(LNDBB) (DBCBB)}

\begin{tabular}{llllll}
\hline DMR10 & $1: 2378997-2379771$ & 1 & 1 & 1 & 1 \\
\hline DMR127 & $3: 10805466-10809820$ & $1.824 \mathrm{e}-2 *$ & $1.078 \mathrm{e}-2^{*}$ & 0.5602 & 1 \\
\hline DMR203 & $6: 28058845-28060341$ & 1 & 1 & $5.064 \mathrm{e}-3^{*}$ & $7.29 \mathrm{e}-$ \\
& & & & $17^{* * *}$ \\
\hline DMR204 & $6: 28833654-28834570$ & 0.997 & 1 & 1 & 1 \\
\hline DMR236 & $7: 1952455-1953065$ & 1 & 1 & 1 & 1 \\
\hline DMR237 & $7: 2048069-2049043$ & 1 & 1 & 1 & $1.367 \mathrm{e}-3^{*}$ \\
\hline DMR291 & $8: 38287269-38289920$ & 0.9733 & 1 & 1 & $1.756 \mathrm{e}-4^{* *}$ \\
\hline DMR526 & $14: 103745950-$ & 1 & - & - & $19 \mathrm{e}-4^{* *}$ \\
\hline DMR527 & $14: 104006646-$ & 1 & & & \\
\hline
\end{tabular}

Table 1 